\title{
MHD duct flows under hydrodynamic "slip" condition
}

Received: 27 October 2008 / Accepted: 23 February 2009 / Published online: 19 May 2009

(C) The Author(s) 2009. This article is published with open access at Springerlink.com

\begin{abstract}
Three classic MHD problems are revisited assuming hydrodynamic slip condition at the interface between the electrically conducting fluid and the insulating wall: (1) Hartmann flow; (2) fully developed flow in a rectangular duct; and (3) quasi-two-dimensional (Q2D) turbulent flow. The first two problems have been solved analytically. Additionally to the Hartmann number $(\mathrm{Ha})$, a new dimensionless parameter $\mathrm{S}$, the ratio of the slip length to the thickness of the Hartmann layer, has been identified. One of the most important conclusions of the paper is that the duct flows with the slip still exhibit Hartmann layers, whose thickness scales as $1 / \mathrm{Ha}$, while the thickness of the side layers is a function of both $\mathrm{Ha}$ and $\mathrm{S}$. In the case of Q2D flows, a new expression for the Hartmann braking time has been derived showing its increase at $H a>>1$ by factor $(1+S)$. Numerical simulations performed for a flow with the "M-shaped" velocity profile show that in the presence of the slip, a Q2D flow becomes more irregular as vortical structures experience less Joule and viscous dissipation in the Hartmann layers.
\end{abstract}

Keywords Magnetohydrodynamic duct flow · Slip condition · Hartmann layer · Quasi-two-dimensional turbulence

PACS 47.65.-d, 47.60.Dx

\section{Introduction}

In most of the cooling applications where a viscous fluid flows in contact with a solid wall, for example in heat exchangers, a common assumption is a good wetability at the liquid-solid interface. When such is the case, the so-called hydrodynamic "no-slip" boundary condition is normally applied. In contrast, in some special applications, e.g., in microfluidic and nanofluidic devices, where the surface-to-volume ratio is large, the slip behavior is more typical, and the mixed "slip" hydrodynamic boundary condition is usually used [1], as first proposed by Navier.

There exist many physical reasons for the slip over hydrophobic surfaces, among which are: molecular slip [2], small dipole moment of polar liquids [3], and trapping the naturally present gas bubbles on the solid surface (see references in [4]). Regardless the slip mechanism, the degree of slip is normally quantified through the slip length $L_{S}$, defined as the distance from the liquid to the surface within the solid phase, where the extrapolated flow velocity vanishes (Fig. 1). The slip length can vary from nanometers [5,6] to microns or even tens of microns for specially designed superhydrophobic surfaces [7]. Using the slip length, the corresponding mixed

Communicated by O. Zikanov

S. Smolentsev

Department of Mechanical and Aerospace Engineering, UCLA, Los Angeles, CA 90095-1597, USA

E-mail: sergey@fusion.ucla.edu 


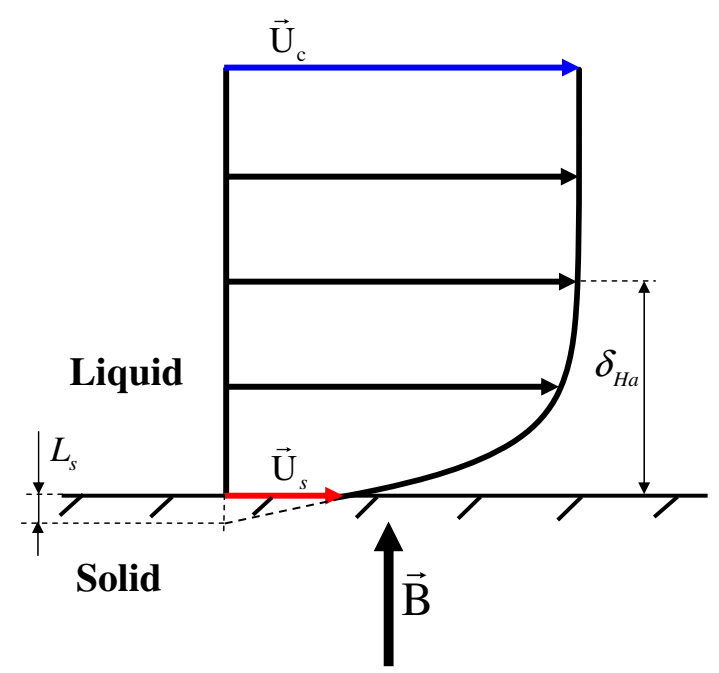

Fig. 1 Sketch of a magnetohydrodynamic flow under hydrodynamic slip conditions

boundary condition at the liquid-solid interface can be written as a linear relation between the slip velocity $U_{s}$ and the tangential stress, which in the case of a simple unidirectional flow, takes the following form:

$$
U_{s}=L_{s} \frac{\mathrm{d} U}{\mathrm{~d} n}
$$

In this paper, we focus on a special case of magnetohydrodynamic (MHD) rectangular duct flows in a uniform transverse magnetic field $B_{0}$, which demonstrate slip behavior (Fig. 1). Without the slip, such flows are known to exhibit special boundary layers: (1) Hartman layers at the walls perpendicular to the magnetic field, whose thickness $\delta_{H a}$ in a strong magnetic field scales as $1 / \mathrm{Ha}$ and (2) the side layers with the thickness scaling as $1 / \sqrt{\mathrm{Ha}}$. Here, the Hartmann number squared $\mathrm{Ha}^{2}$ expresses the ratio between the electromagnetic and the viscous force in the fluid: $H a=B_{0} b \sqrt{\sigma / v \rho}$, where $b$ is the Hartmann length (half of the duct width in the magnetic field direction), and $\sigma, v$, and $\rho$ are the fluid electrical conductivity, kinematic viscosity, and the density correspondingly. In strong magnetic field applications (e.g., liquid-metal blankets of a fusion reactor), $H a \sim 10^{3}-10^{5}$. In these conditions, if the slip behavior occurs, the slip length can be comparable or even larger than the thickness of the Hartmann layer, indicating that the slip effect on MHD flows can be much more pronounced compared to ordinary flows, where the boundary layer is typically much thicker. For example, for a $10 \mathrm{~T}$ magnetic field typical to future fusion reactors, the estimate for the Hartmann layer thickness in a liquid metal flow is $1 \mu \mathrm{m}$. One can expect a pronounced slip effect on such a flow if $L_{S} / \delta_{H a}>0.1$ that corresponds to the conditions when $L_{S}>100 \mathrm{~nm}$. Providing the micron size slip length could exist, the corresponding ratio of the slip length to the Hartmann layer thickness would exceed unity. This value, as shown below, would result in a dramatic effect on the blanket flows.

In liquid flows, the slip length can be affected by many physico-chemical parameters, including wetting, surface roughness, impurities, dissolved gas, shear rate, pressure, temperature, etc. [6]. The physical mechanism of the interfacial slip is interplay of all these parameters. Among them, wetting is always considered to be the most important factor as almost all experiments on hydrophobic surfaces have indicated some drag reduction, often demonstrating strong correlation between the slip length and the contact angle (see references in [6]). Another key parameter is the surface roughness, which can either decrease or increase the slip length. The increase can be associated with the increase of contact area and especially with trapping gas bubbles, which are usually present in the liquid. The effect of poor wetting in MHD flows between liquid metals and metallic walls are well known among experimentalists. However, running the liquid metal flow for a sufficiently long time eliminates this effect due to formation of an inter-metallic layer. As for ceramics, it seems to be different, as no chemical interaction occurs with liquid metals even at elevated temperatures. As an example, we can refer to experimental studies for the eutectic alloy lead-lithium ( $\mathrm{PbLi}$ ) in contact with silicon carbide ( $\mathrm{SiC})$, which have demonstrated poor wetting over a long period of time [8-10]. One can anticipate similar behavior in the so-called DCLL (dual-coolant lead-lithium) blanket [11], where PbLi flows occur inside the electroinsulating flow insert made of $\mathrm{SiC}$, either composite or foam. Additional factors, which most likely can 
increase the slip length in DCLL-type blankets, are formation of the helium bubbles in the PbLi and the wall roughness associated with the structure of silicon carbide.

In what follows, we assume conditions leading to the interfacial slip with the slip length constant over the interface area. The relevant MHD flow equations are essentially the same as standard ones (see, e.g., [12]) and are not duplicated here. The solid structure confining the fluid is assumed to be electrically non-conducting, so that all induced currents are closed in the flow domain. Under these assumptions, we readdress three classic MHD problems by replacing the no-slip hydrodynamic boundary condition with the slip condition in the form suggested by Eq. (1). The problems considered below are analogs for: (1) Hartmann flow [13], (2) fully developed laminar flow in a rectangular duct [14], and (3) quasi-two-dimensional (Q2D) turbulent flow in a strong magnetic field [15].

\section{Hartmann flow with the slip}

Hartmann flow is the MHD counterpart of the classic plane Poiseuille flow [13]. In this problem, an electrically conducting liquid flows in the $x$ direction between two parallel walls, which are $2 b$ apart. The magnetic field is applied in the $z$ direction, perpendicular to the walls. The axial velocity $U(z)$ and the induced magnetic field $b_{x}(z)$ are governed by two equations (see, e.g., [16]):

$$
\begin{aligned}
& 0=1+\frac{\mathrm{d}^{2} \tilde{U}}{\mathrm{~d} \tilde{z}^{2}}+H a \frac{\mathrm{d} \tilde{b}_{x}}{\mathrm{~d} \tilde{z}}, \\
& 0=\frac{\mathrm{d}^{2} \tilde{b}_{x}}{\mathrm{~d} \tilde{z}^{2}}+H a \frac{\mathrm{d} \tilde{U}}{\mathrm{~d} \tilde{z}} .
\end{aligned}
$$

Here, the equations are written in a dimensionless form as indicated by " ". The velocity scale is built using the flow-driving pressure gradient $\mathrm{d} P / \mathrm{d} x:[U]=b^{2} v^{-1} \rho^{-1}(-\mathrm{d} P / \mathrm{d} x)$; the induced magnetic field is scaled by $\left[b_{x}\right]=[U] \mu_{0} \sqrt{\sigma v \rho}$ ( $\mu_{0}$ is the magnetic permeability); and the Hartmann length $b$ is used as the length scale. The boundary conditions in the presence of the slip are the following:

$$
\tilde{z}= \pm 1: \tilde{U} \pm \alpha \frac{d \tilde{U}}{d \tilde{z}}=0, \quad \tilde{b}_{x}=0,
$$

where $\alpha=L_{s} / b$ denotes the dimensionless slip length. The solution to this problem is as follows:

$$
\begin{aligned}
& \tilde{U}(\tilde{z})=\alpha+\frac{1}{H a \tanh (H a)}\left(1-\frac{\cosh (H a \tilde{z})}{\cosh (H a)}\right), \\
& \tilde{b}_{x}(\tilde{z})=-\frac{\tilde{z}}{H a}+\frac{1}{H a \tanh (H a)} \frac{\sinh (H a \tilde{z})}{\cosh (H a)} .
\end{aligned}
$$

As a matter of fact, the only difference of this solution with the classic Hartmann solution is the term $\alpha$ in formula (5) for the velocity. As a result, the thickness of the Hartmann layer does not depend on $L_{s}$ : in a strong magnetic field, $\delta_{\mathrm{Ha}} \sim 1 / \mathrm{Ha}$. Therefore, the structure of the Hartmann flow in the slip conditions is still similar to that without the slip. Namely, the flow demonstrates a uniform core and two thin Hartmann layers. The velocity in the core is, however, different. In a strong magnetic field, with the slip, $\tilde{U}_{c}=\alpha+1 / \mathrm{Ha}$, while $\tilde{U}_{c}=1 / H a$ in the no-slip conditions. At the same time, the induced magnetic field distribution in the core does not change with the slip: $\tilde{b}_{c}=-\tilde{z} / H a$.

It is convenient to rescale solution (5) using the mean bulk velocity $U_{m}=\frac{1}{2 b} \int_{-b}^{b} U(z) \mathrm{d} z$ as a new scale. After integrating Eq. (5),

$$
\tilde{U}_{m}=\alpha+\frac{1}{H a \tanh (H a)}\left(1-\frac{\tanh (H a)}{H a}\right)
$$

and

$$
\frac{U}{U_{m}}=\frac{H a}{H a(1+S)-\tanh (H a)}\left(1+S-\frac{\cosh (H a \tilde{z})}{\cosh (H a)}\right)
$$



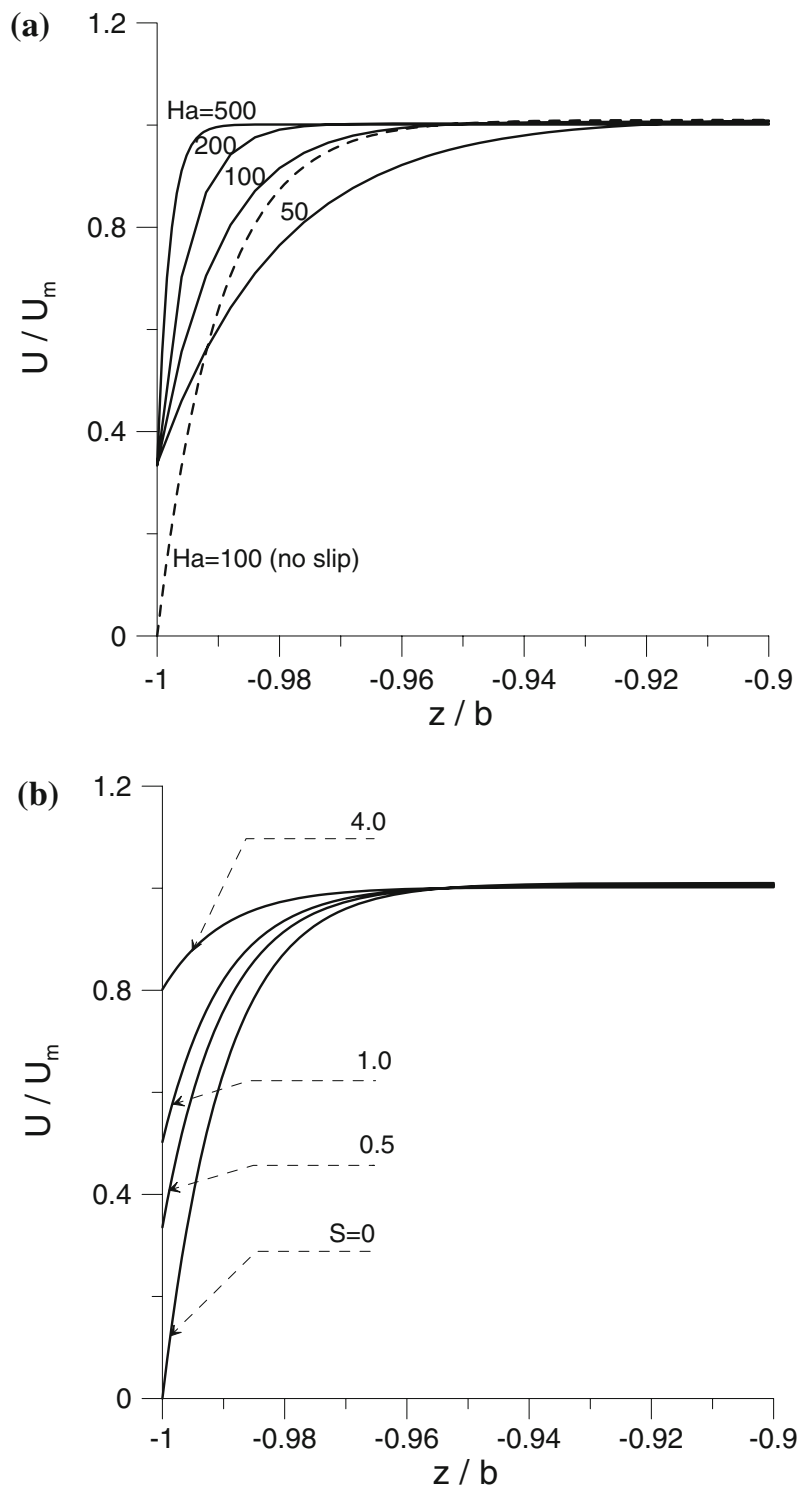

Fig. 2 Velocity distribution in the Hartmann layer in the 1-D flow with the slip: a effect of $\mathrm{Ha}$ at $S=0.5$ and $\mathbf{b}$ effect of $S$ at $H a=100$

where

$$
S=\alpha H a \tanh (H a)
$$

Therefore, the velocity profile in the Hartmann flow under the slip conditions is fully determined by two parameters: $H a$ and $S$ (Fig. 2).

Two useful formulas can be derived from Eq. (8). First, the slip to mean bulk velocity ratio is

$$
\frac{U_{s}}{U_{m}}=\frac{S H a}{H a(1+S)-\tanh (H a)} .
$$

Second, the pressure drop coefficient, defined as $\lambda=-\frac{\mathrm{d} P / \mathrm{d} x}{\rho U_{m}^{2} / 2 b}$, is

$$
\lambda=2 \frac{H a}{R e} \frac{H a \tanh (H a)}{H a(1+S)-\tanh (H a)},
$$


where $R e=U_{m} b / v$ is the Reynolds number. This formula can be rewritten using $U_{s} / U_{m}$ and $S$ as

$$
\lambda=2 \frac{H a}{\operatorname{Re}} \frac{U_{s}}{U_{m}} \frac{1}{S} \tanh (H a) .
$$

In the asymptotic case of high Hartmann numbers, Eqs. (9), (10), and (12) can be simplified:

$$
\begin{aligned}
S & =\frac{L_{s}}{\delta_{H a}}, \\
\frac{U_{s}}{U_{m}} & =\frac{S}{1+S}, \\
\lambda & =\frac{\lambda_{H a}}{1+S} .
\end{aligned}
$$

Equation (13) allows a very simple interpretation of parameter $S$ as the ratio between the slip length and the Hartmann layer thickness. Equation (14) shows that in a strong magnetic field, the slip to mean velocity ratio is fully determined by parameter $S$. Formula (15) illustrates directly the reduction of the pressure drop coefficient in the flow under the slip conditions compared to that $\lambda_{\mathrm{Ha}}=2 \mathrm{Ha} / \mathrm{Re}$ in the classic Hartmann flow in a strong magnetic field.

The following example stresses the significance of the slip in blanket applications. Typically, in a liquid metal blanket, $b=0.1 \mathrm{~m}, H a=10^{4}$ (at $B_{0} \sim 5 \mathrm{~T}$ ), so that $\delta_{H a}=10 \mu \mathrm{m}$. Providing $L_{s}=10 \mu \mathrm{m}$, the anticipated reduction in the MHD pressure drop coefficient due to the interfacial slip based on Eq. (15) is by factor of two. Measuring the pressure drop reduction in experimental conditions would be challenging unless strong multi-Tesla magnetic fields, comparable with those in the fusion reactor, are used. Finally, it should be noted, that the strong effect of the slip on the pressure drop in the Hartmann flow is due to the fact that the slip length, even small, can be comparable with the thickness of the Hartmann layer, which is, as shown here, not affected by the slip itself.

\section{Fully developed flow in a rectangular duct with the slip at the Hartmann walls and no slip at the side walls}

We consider a fully developed flow in a rectangular duct (Fig. 3) driven by a pressure gradient. The Hartmann walls are treated as "slip" walls, while the other two (the side walls) are assumed to have no slip. Relative to

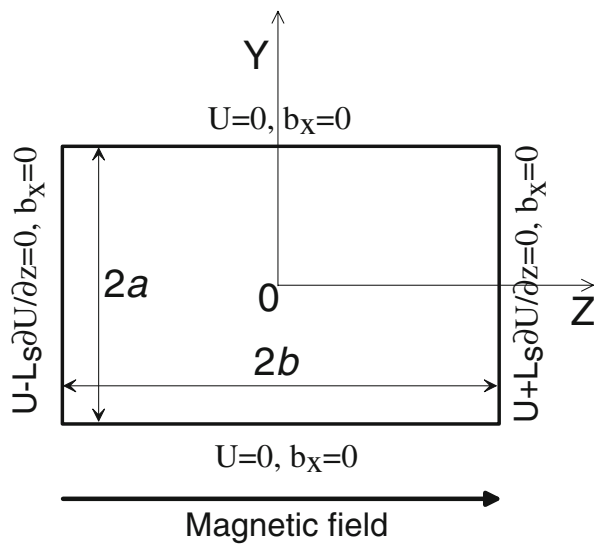

Fig. 3 Sketch of the 2-D reference duct flow with the slip 
the flow sketch in Fig. 3, the equations describing the flow are (see, e.g. [14]):

$$
\begin{aligned}
& \frac{\partial^{2} \tilde{U}}{\partial \tilde{z}}+\frac{\partial^{2} \tilde{U}}{\partial \tilde{y}}+1+H a \frac{\partial \tilde{b}_{x}}{\partial \tilde{z}}=0, \\
& \frac{\partial^{2} \tilde{b}_{x}}{\partial \tilde{z}^{2}}+\frac{\partial^{2} \tilde{b}_{x}}{\partial \tilde{y}^{2}}+H a \frac{\partial \tilde{U}}{\partial \tilde{z}}=0 .
\end{aligned}
$$

These equations are written in the dimensionless form, using the same scales as described above. The boundary conditions at the Hartmann walls $\tilde{z}= \pm 1$ and at the side walls $\tilde{y}= \pm d(d=a / b)$ are the following:

$$
\begin{aligned}
& \tilde{z}= \pm 1: \tilde{U} \pm \alpha \frac{\partial \tilde{U}}{\partial \tilde{z}}=0, \quad \tilde{b}_{x}=0 \\
& \tilde{y}= \pm d: U=0, \quad b_{x}=0 .
\end{aligned}
$$

It should be mentioned that the present choice of boundary conditions (namely, there is slip only at the Hartmann walls) is based only on our intention to obtain a purely analytical solution using a standard technique of expanding the velocity and the induced magnetic field as Fourier cosine series in the direction perpendicular to the applied magnetic field. More general case (all walls have the slip) cannot be attacked in the same way because of the limitations of this analytical technique and will require a numerical or asymptotic approach. The main conclusion about the modification of the side layer thickness (see the end of this section) seems, however, to be qualitatively the same in both cases.

We look for solution of Eqs. (16) and (17) in the form of the expansion of the velocity and the induced magnetic field as Fourier cosine series in $\tilde{y}$ :

$$
\tilde{U}(\tilde{z}, \tilde{y})=\sum_{n=0}^{\infty} f_{n}(\tilde{z}) \cos \left(a_{n} \tilde{y}\right), \quad \tilde{b}_{x}(\tilde{z}, \tilde{y})=\sum_{n=0}^{\infty} g_{n}(\tilde{z}) \cos \left(a_{n} \tilde{y}\right) .
$$

To satisfy boundary conditions (19), one should put $a_{n}=\left(n+\frac{1}{2}\right) \frac{\pi}{d}$. Inserting series (20) into Eqs. (16) and (17), and using the identity $1=\frac{4}{\pi} \sum_{n=0}^{\infty} \frac{(-1)^{n}}{(2 n+1)} \cos \left(a_{n} \tilde{y}\right)$, one can find the following ordinary differential equations for unknown functions $f_{n}$ and $g_{n}(n=0,1,2 \ldots)$ :

$$
f_{n}^{\prime \prime}-a_{n}^{2} f_{n}+\frac{4}{\pi} \frac{(-1)^{n}}{(2 n+1)}+H a g_{n}^{\prime}=0, \quad g_{n}^{\prime \prime}-a_{n}^{2} g_{n}+H a f_{n}^{\prime}=0 .
$$

The boundary conditions are obtained from original boundary conditions (18) as follows:

$$
z= \pm 1: f_{n} \pm \alpha f_{n}^{\prime}=0, \quad g_{n}=0 .
$$

Taking into account symmetry in $U$ and asymmetry in $b_{x}$, the solution of Eqs. (21) with boundary conditions (22) is sought as

$$
f_{n}=k_{n}+A_{n} \cosh \left(\alpha_{n} \tilde{z}\right)+B_{n} \cosh \left(\beta_{n} \tilde{z}\right), \quad g_{n}=A_{n} \sinh \left(\alpha_{n} \tilde{z}\right)+B_{n} \sinh \left(\beta_{n} \tilde{z}\right) .
$$

Substituting (23) into (21) results in two equations, which determine $k_{n}, \alpha_{n}$ and $\beta_{n}$ :

$$
\begin{aligned}
& -a_{n}^{2} k_{n}+\frac{4}{\pi} \frac{(-1)^{n}}{(2 n+1)}=0, \\
& A_{n}\left(\alpha_{n}^{2}+H a \alpha_{n}-a_{n}^{2}\right)+B_{n}\left(\beta_{n}^{2}+H a \beta_{n}-a_{n}^{2}\right)=0 .
\end{aligned}
$$

From Eq. (24), one can find

$$
k_{n}=\frac{2(-1)^{n}}{a_{n}^{3} d}
$$

From Eq. (25),

$$
\alpha_{n}=\frac{-H a+\sqrt{H a^{2}+4 a_{n}^{2}}}{2}, \quad \beta_{n}=\frac{-H a-\sqrt{H a^{2}+4 a_{n}^{2}}}{2} .
$$




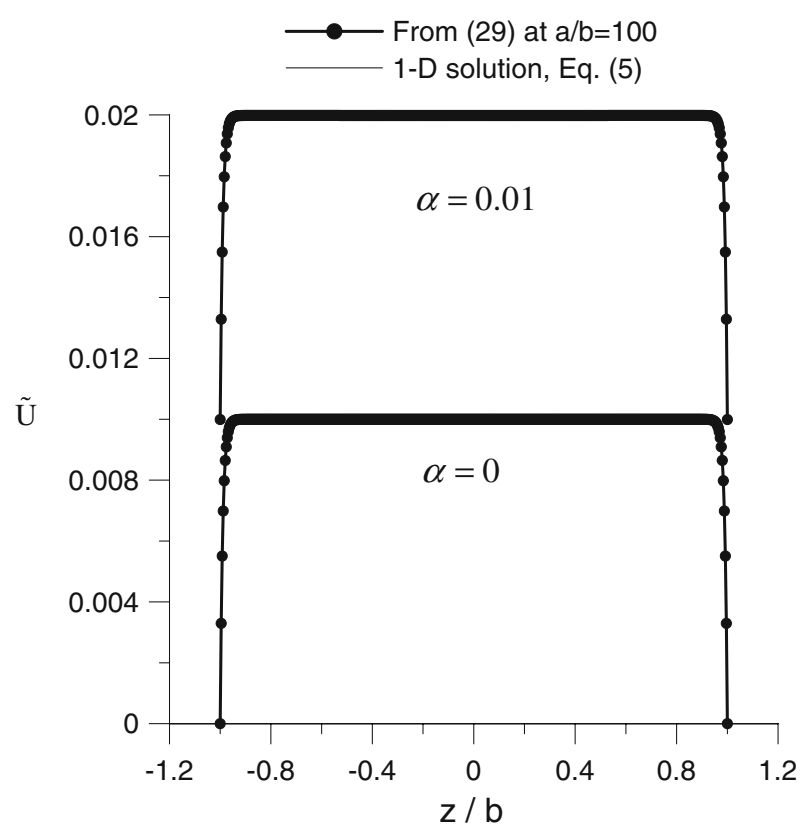

Fig. 4 Comparison between 1-D (Eq. 5) and 2-D solution (Eqs. 29) for a slotted duct ( $d=100)$ at $H a=100$

Two constants $A_{n}$ and $B_{n}$ entering solution (23) can be obtained from boundary conditions (22). They are

$$
\begin{aligned}
A_{n} & =\frac{-k_{n}}{\cosh \left(\alpha_{n}\right)-\sinh \left(\alpha_{n}\right) \operatorname{coth}\left(\beta_{n}\right)+\alpha\left(\alpha_{n}-\beta_{n}\right) \sinh \left(\alpha_{n}\right)}, \\
B_{n} & =\frac{-k_{n}}{\cosh \left(\beta_{n}\right)-\sinh \left(\beta_{n}\right) \operatorname{coth}\left(\alpha_{n}\right)+\alpha\left(\beta_{n}-\alpha_{n}\right) \sinh \left(\beta_{n}\right)} .
\end{aligned}
$$

After collecting all results together, the dimensionless velocity and the induced magnetic field in the rectangular duct with the slip at the Hartmann walls and no slip at the side walls are given by

$$
\left\{\begin{array}{l}
\tilde{U}=\sum_{n=0}^{\infty} k_{n} \cos \left(a_{n} \tilde{y}\right)\left[1-\frac{\cosh \left(\alpha_{n} \tilde{z}\right)}{\cosh \left(\alpha_{n}\right)-\sinh \left(\alpha_{n}\right) \operatorname{coth}\left(\beta_{n}\right)+\alpha\left(\alpha_{n}-\beta_{n}\right) \sinh \left(\alpha_{n}\right)}-\frac{\cosh \left(\beta_{n} \tilde{z}\right)}{\cosh \left(\beta_{n}\right)-\sinh \left(\beta_{n}\right) \operatorname{coth}\left(\alpha_{n}\right)+\alpha\left(\beta_{n}-\alpha_{n}\right) \sinh \left(\beta_{n}\right)}\right] \\
\tilde{b}_{x}=-\sum_{n=0}^{\infty} k_{n} \cos \left(a_{n} \tilde{y}\right)\left[\frac{\sinh \left(\beta_{n} \tilde{c}\right)}{\cosh \left(\alpha_{n}\right)-\sinh \left(\alpha_{n}\right) \operatorname{coth}\left(\alpha_{n} \tilde{z}\right)+\alpha\left(\alpha_{n}-\beta_{n}\right) \sinh \left(\alpha_{n}\right)}+\frac{\cosh \left(\beta_{n}\right)-\sinh \left(\beta_{n}\right) \operatorname{coth}\left(\alpha_{n}\right)+\alpha\left(\beta_{n}-\alpha_{n}\right) \sinh \left(\beta_{n}\right)}{\cos }\right] \\
a_{n}=\left(n+\frac{1}{2}\right) \frac{\pi}{d}, k_{n}=\frac{2(-1)^{n}}{a_{n}^{3} d} \\
\alpha_{n}=\frac{-H a+\sqrt{H a^{2}+4 a_{n}^{2}}}{2}, \beta_{n}=\frac{-H a-\sqrt{H a^{2}+4 a_{n}^{2}}}{2}
\end{array}\right.
$$

It can be demonstrated that solution (29) reduces to the solution of the Hartmann problem with the slip obtained in Sect. 2, providing $d \rightarrow \infty$ (Fig. 4). It also reduces to the quasi 2-D Poiseuille flow in case of large $\alpha$.

If the dimensionless volumetric flow rate, $\tilde{Q}$, is defined by $\tilde{Q}=\int_{-d}^{d} \mathrm{~d} \tilde{y} \int_{-1}^{1} \tilde{U}(\tilde{z}, \tilde{y}) \mathrm{d} \tilde{z}$, then, from (29),

$$
\begin{aligned}
\tilde{Q}=4 \sum_{n=0}^{\infty} \frac{k_{n}}{a_{n}} \sin \left(a_{n} d\right)[ & 1-\frac{\sinh \left(\alpha_{n}\right) / \alpha_{n}}{\cosh \left(\alpha_{n}\right)-\sinh \left(\alpha_{n}\right) \operatorname{coth}\left(\beta_{n}\right)+\alpha\left(\alpha_{n}-\beta_{n}\right) \sinh \left(\alpha_{n}\right)} \\
& \left.-\frac{\sinh \left(\beta_{n}\right) / \beta_{n}}{\cosh \left(\beta_{n}\right)-\sinh \left(\beta_{n}\right) \operatorname{coth}\left(\alpha_{n}\right)+\alpha\left(\beta_{n}-\alpha_{n}\right) \sinh \left(\beta_{n}\right)}\right] .
\end{aligned}
$$

The pressure drop coefficient defined in Sect. 2 can easily be calculated using $\tilde{Q}$ as $\lambda=\frac{8}{\operatorname{Re}} \frac{1}{\tilde{Q}}$. Its behavior is illustrated in Fig. 5 for a square duct $(d=1)$ as a function of $H a$ and $\alpha$. 


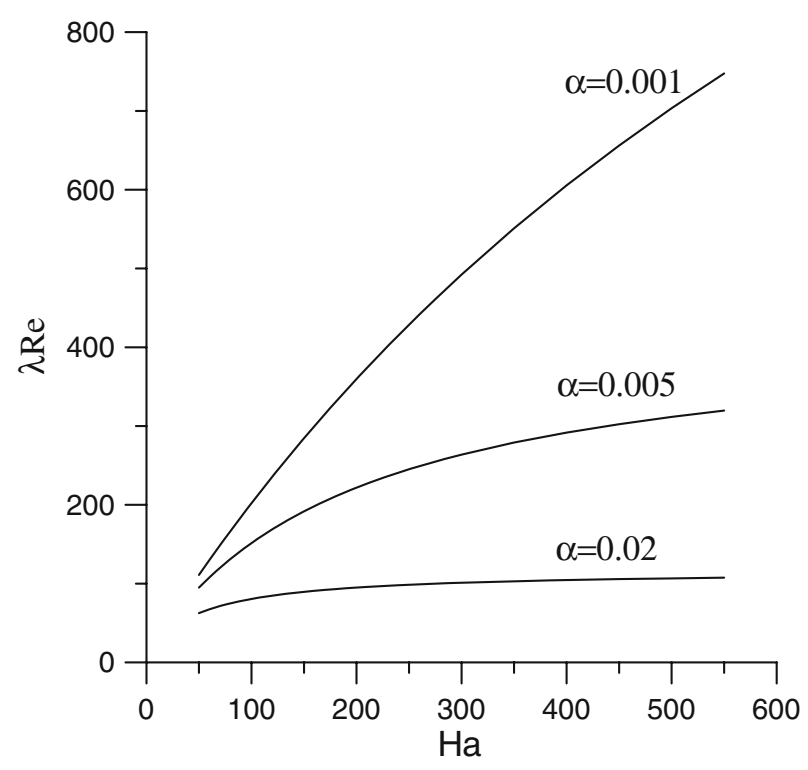

Fig. 5 Effect of the slip and Hartmann number on the pressure drop coefficient in a square duct

Figure 6 illustrate the slip effect on the velocity profile and the induced magnetic field distribution. One can see that similar to the classic problem without the slip, the flow with the slip exhibits a core, where the velocity tends to be uniform, while the velocity gradients are confined to the boundary layers. The boundary layers at the walls perpendicular to the magnetic field, as directly seen from the numerical data, still have the thickness scaling as $1 / \mathrm{Ha}$ and thus can be referred to as Hartmann layers. This conclusion also matches the analysis in Sect. 2 for the particular case of the 1-D Hartmann flow with the slip, where, as shown, the thickness of the boundary layer is not affected by the slip. The boundary layers at the side walls in the 2-D case are, however, not scaled as $1 / \sqrt{\mathrm{Ha}}$ (Fig. 7). They can be significantly thicker than the classic side layers, especially if the slip length is comparable or larger than the thickness of the Hartmann layer. Correspondingly, the core area of uniform velocity in the center of the duct can be significantly smaller compared to the same flow in the no-slip conditions. There are also four regions at the corners where the Hartmann and the side layers overlap.

Notice that the dimensionless slip length $\alpha$ enters both the series for the velocity in (29) and Eq. (30) for the flow rate through the term $\alpha\left(\alpha_{n}-\beta_{n}\right)$ in the denominator, which at high $\mathrm{Ha}$ transforms to $S=L_{S} / \mathrm{Ha}$. Therefore, similar to the 1-D case, the reference fully developed 2-D flow in a strong magnetic field is also fully determined by the two parameters: $H a$ and $S$. Based on the results obtained, it appears that parameter $S$ plays a very important role in both the 1-D (Hartmann flow) and the 2-D problem. Namely, it determines the relative contribution of the MHD effect and the slip itself. In the strong slip conditions, when $S>>1$, the induced electric currents are small, having almost no effect on the flow, and the flow is fully controlled by the slip phenomenon. On the contrary, if $S<<1$, the flows don't differ much from the corresponding classic MHD flows without the slip. In the situations when $S$ is of the order of unity, both MHD and slip effects are important.

\section{Quasi-two-dimensional MHD turbulent flow with the slip at the Hartmann walls}

Here, we consider a flow of an electrically conducting fluid in a rectangular duct under the influence of a strong, transverse, externally applied magnetic field. The cross-section of the duct and the magnetic field orientation are sketched in Fig. 3 (see Sect. 3). The flow is assumed to be non-uniform in the $x$ (axial) direction and may demonstrate unsteady or even turbulent behavior as the liquid proceeds downstream. Such a flow, under the no-slip conditions, has been intensively studied for decades in the context of Q2D turbulence (see, e.g., [15]). The novelty introduced in the present paper is the slip condition. In doing so, the Hartmann walls are considered as slip walls while the other two walls are assumed to have no slip in accordance with boundary conditions (18) and (19). 

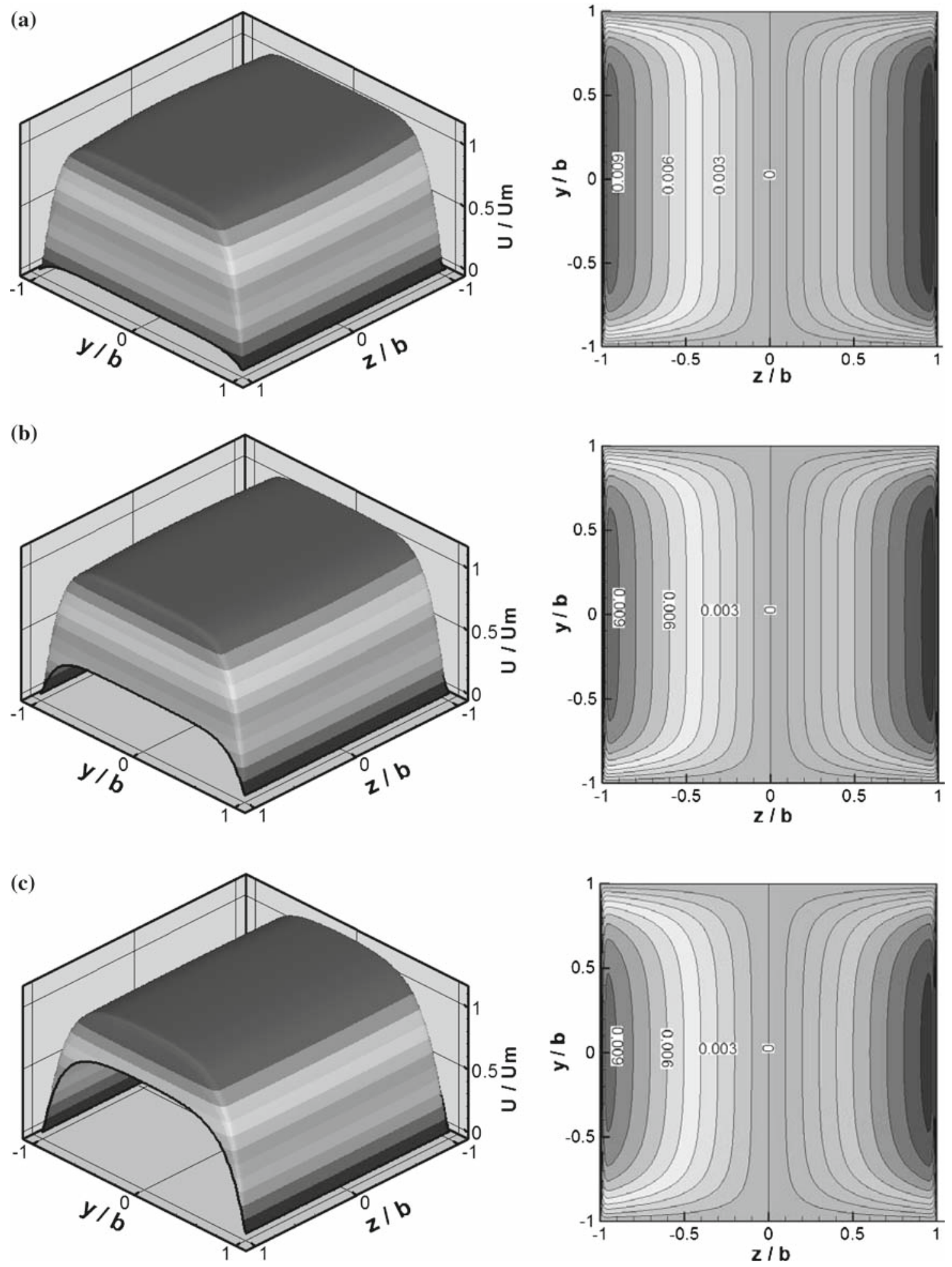

Fig. 6 Effect of the slip on the velocity (left) and the induced magnetic field $\tilde{b}_{x}$ (right) in a square duct at $H a=100$ : $\mathbf{a} \alpha=0.001, S=0.1 ; \mathbf{b} \alpha=0.005, S=0.5 ; \mathbf{c} \alpha=0.02, S=2$ 


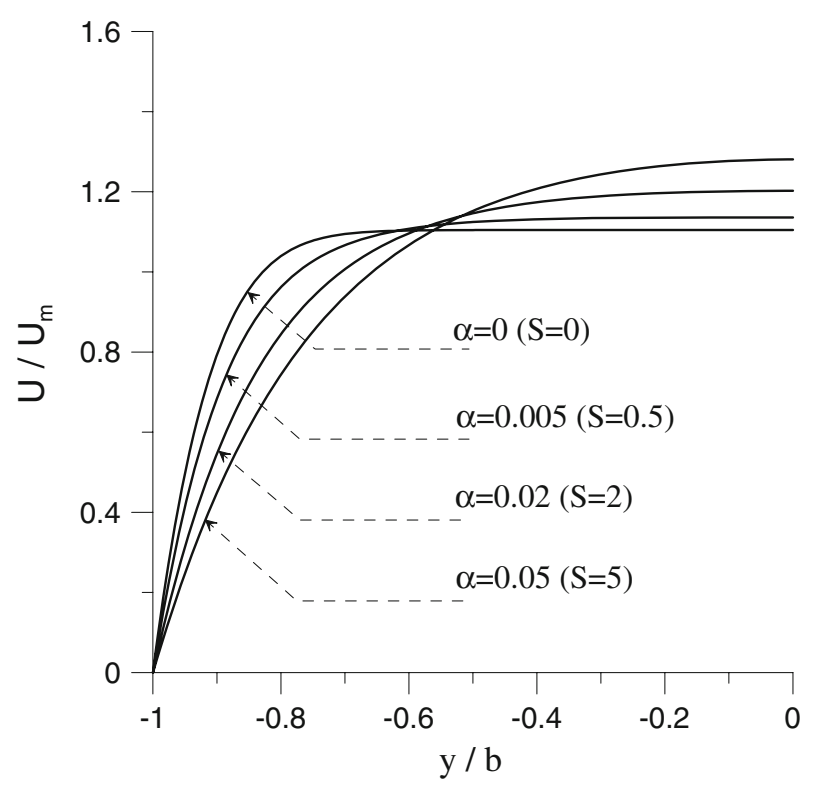

Fig. 7 Effect of the slip on velocity distribution in the side layer $(y=0)$ in a square duct at $H a=100$

The underlying physics of MHD duct flows in a strong magnetic field is related to the predominant electromagnetic dissipation mechanism (Joule dissipation), which enforces a strong flow anisotropy, until the limit of Q2D turbulence is achieved. In a Q2D flow, the turbulence structures appear as big (comparable in size to the duct dimension) columnar-like vortices with their axis aligned with the field direction. Three-dimensional features can still be observed in the thin Hartmann layers, where almost all ohmic and viscous losses occur, while the influence of inertia is negligible. Such Q2D eddies do not induce much electric current and thus are weakly affected by the magnetic field. They persist over many eddy turnovers, until being damped via slow dissipating processes in the Hartmann layers.

The major impact of the slip on the Q2D flow is the reduction of the dissipation losses in the Hartmann layers, which still exist, as seen directly from the analysis in Sects. 2 and 3. To incorporate this new feature in the mathematical model, we follow the general approach, first proposed in [15], by integrating the original 3-D flow equations along the magnetic field lines. When doing so, the velocity components in such a Q2D flow are presented in the following way:

$$
U=-\frac{\partial \psi}{\partial y} f(z), \quad V=\frac{\partial \psi}{\partial x} f(z), \quad W=0,
$$

where $\psi(x, y)$ is the 2-D stream function. The function $f$, independent on $x$ and $y$, describes the variations of the two flow components $U$ and $V$ in the direction of the applied magnetic field. In accordance with the present analysis in Sects. 2 and 3, this function has the following form:

$$
f(z)=\frac{1}{1+S}\left(1+S-e^{H a(|z / b|-1)}\right) \text { for } H a>>1 .
$$

After inserting (31) and (32) in the original 3-D MHD equations and integrating them in $z$ between the Hartmann walls (for details of integration, see, e.g. [17]), one ends up with the following set of 2-D equations written in terms of the stream function and the $z$-component of vorticity $\left(\omega(x, y)=(\nabla \times \mathbf{V})_{z}\right)$ :

$$
\begin{gathered}
\frac{\partial \omega}{\partial t}-\frac{\partial \psi}{\partial y} \frac{\partial \omega}{\partial x}+\frac{\partial \psi}{\partial x} \frac{\partial \omega}{\partial y}=v\left(\frac{\partial^{2} \omega}{\partial x^{2}}+\frac{\partial^{2} \omega}{\partial y^{2}}\right)-\frac{\omega}{\tau}, \\
\frac{\partial^{2} \psi}{\partial x^{2}}+\frac{\partial^{2} \psi}{\partial y^{2}}=\omega .
\end{gathered}
$$

An additional linear-drag term $\omega / \tau$ on the right-hand side of Eq. (33) models the damping of Q2D vortices at the Hartmann walls. This term includes a new parameter $\tau$, called Hartmann braking time, which is a 


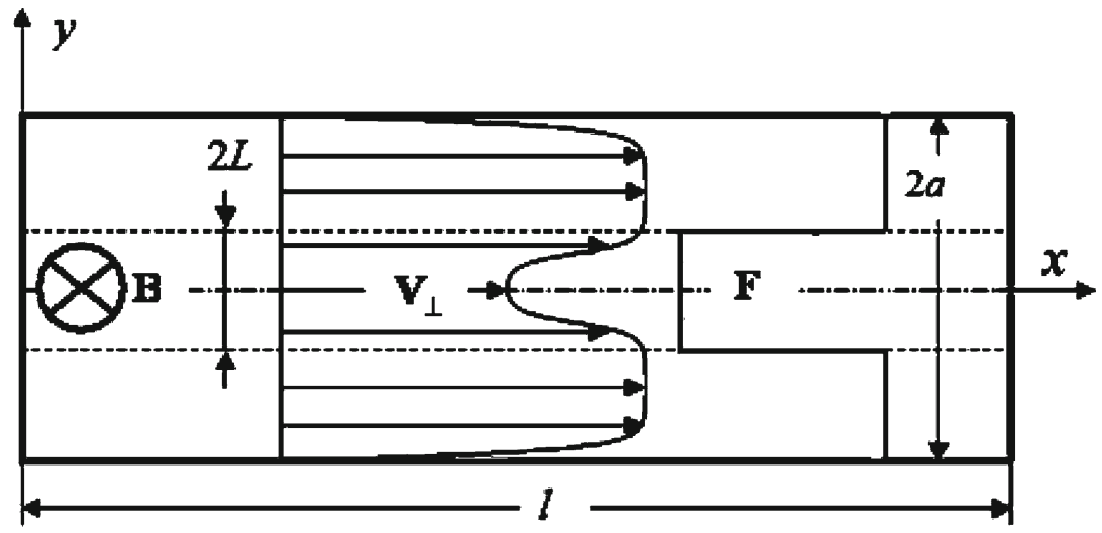

Fig. 8 Sketch of the mid-plane of the Q2D turbulent MHD flow in a rectangular duct

characteristic timescale of vortex damping due to dissipation losses in the Hartmann layers:

$$
\tau=\frac{1+S}{H a} \frac{b^{2}}{v}=(1+S) \tau_{0}
$$

The only difference of the newly-derived parameter $\tau$ with the classic Hartmann braking time $\tau_{0}=\frac{1}{H a} \frac{b^{2}}{\nu}$ (see, e.g. [15]) is the additional factor $(1+S)$, which expresses the increase in the Hartmann braking time associated with the reduction of dissipation losses at the Hartmann walls due to the slip.

To illustrate the slip effect on a Q2D flow, numerical simulations are performed for a flow sketched in Fig. 8. The flow is driven by a pressure gradient and is opposed by a given uniform volumetric force $\mathbf{F}\left(-F_{x}, 0,0\right)$ applied over the whole duct length $l$ :

$$
F_{x}= \begin{cases}F_{0} & \text { for }-L \leq y \leq L \\ 0 & \text { for }-a \leq y<-L, L<y \leq a .\end{cases}
$$

Other details of the mathematical model and numerical procedure are explained in [18], where similar computations were performed under the no-slip condition. The reference flow can be considered as a plane model of the experimental flow in [19], where the flow opposing force appears due to applied voltage between the row of internal electrodes embedded in the Hartmann wall and the cylindrical flow confining wall as the second electrode. Providing the voltage is applied between the two rows of internal electrodes, the volumetric force in this experiment would be very similar to that in the reference hypothetic flow (private communication with Karim Messadek). Realization of such an experiment in practice could, however, be difficult and is not discussed here as the main goal is to illustrate the anticipated effect. The problem with the slip is fully determined by the following dimensionless numbers: the Reynolds number $R e=U_{m} a / v$, the Hartmann number $H a=B_{0} b \sqrt{\sigma / v \rho}$, the modified Froude number $F r=U_{m}^{2} / F_{0} a$, the duct aspect ratio $a / b$, and the slip length to the Hartmann layer thickness ratio $S=L_{S} / \delta_{H a}$. The last parameter is specific to the problem with the slip. In the computations, $S$ is changed from 0 to 1 , while other parameters remain constant. The reference flow exhibits inflection points and at sufficiently high Reynolds numbers becomes unstable and eventually turbulent. The effect of the slip on the flow is illustrated in Figs. 9 and 10. As expected, as parameter $S$ increases, the fluctuations associated with the inflectional instability increase in amplitude and the flow becomes more irregular.

\section{Summary and concluding remarks}

In this paper, the classic problem for MHD flow in an insulating rectangular duct has been extended to take into consideration the hydrodynamic slip at the interface between the flowing liquid and the solid wall. The slip effect is introduced through the boundary condition in the form of Eq. (1), first proposed by Navier, which relates the slip velocity and the tangential stress at the interface via the slip length. As current experimental data suggest [8-10], the slip in MHD flows will likely occur in those fusion applications where eutectic alloy PbLi flows in contact with silicon carbide. Although the slip effect is usually negligibly small in ordinary 

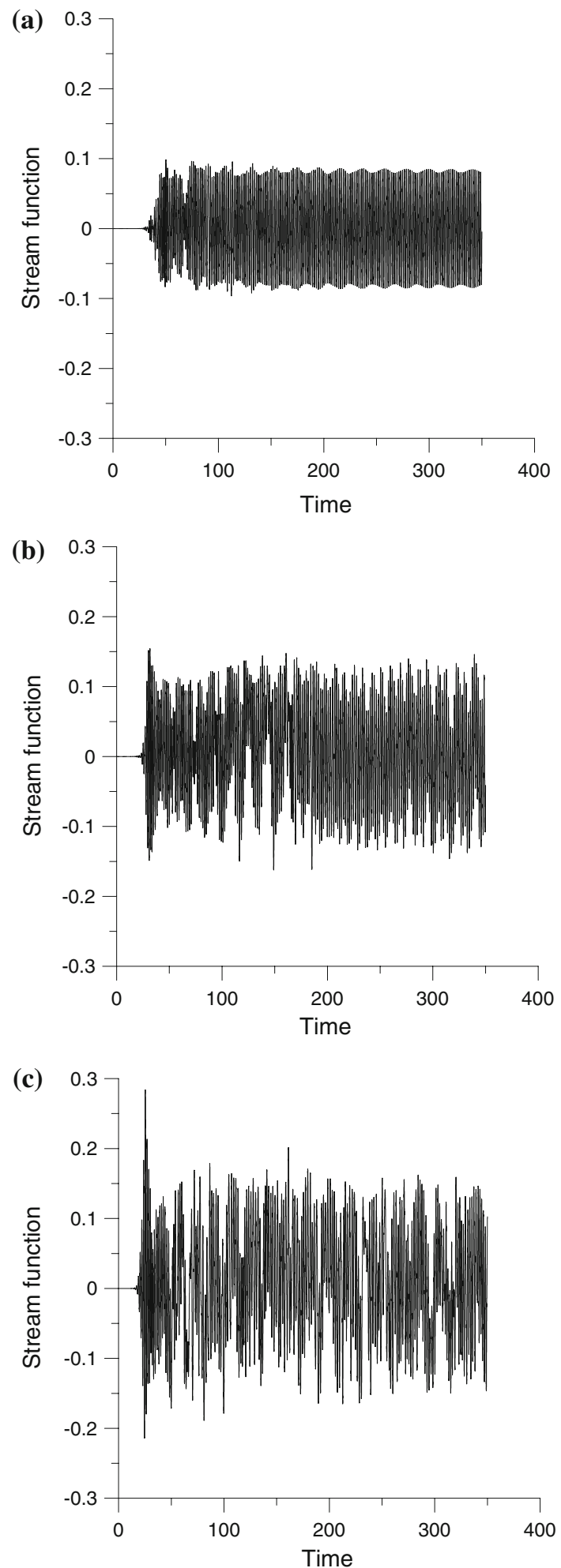

Fig. 9 Fluctuations of the stream function (at $x / a=10, y / a=0$ ) in the flow with the slip at $\operatorname{Re}=1,000, \mathrm{Ha}=100, \mathrm{Fr}=$ $10, a / b=1, L / a=0.4: \mathbf{a} S=0, \mathbf{b} S=0.5, \mathbf{c} S=1$ 

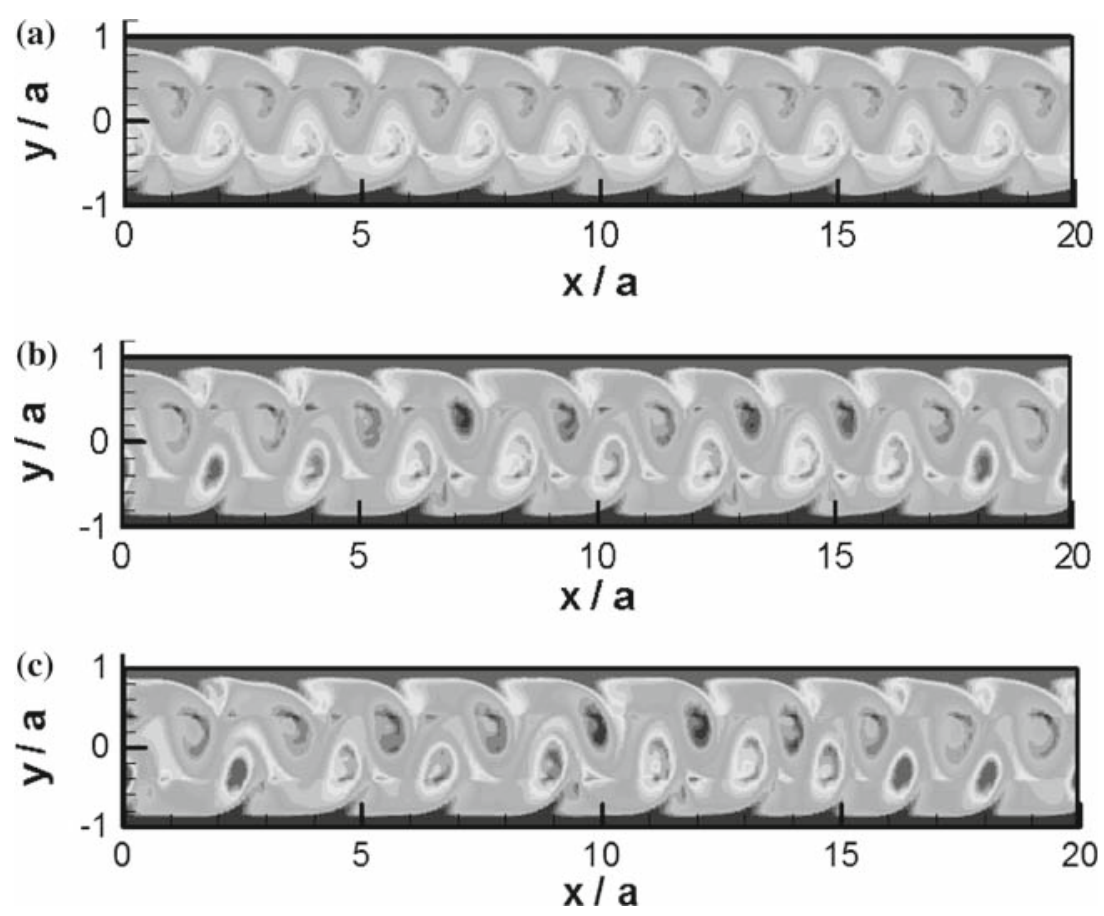

Fig. 10 Effect of the slip on the vorticity distribution in the Q2D flow at $\operatorname{Re}=1,000, H a=100, F r=10, a / b=1, L / a=0.4$ : a $S=0, \mathbf{b} S=0.5, \mathbf{c} S=1$

flows, in MHD flows, it appears to be much more important, since the slip length can be comparable or even larger than the thickness of the Hartmann layer.

First, the Hartmann problem with the slip has been solved analytically to characterize the slip effect on the Hartmann layer. Although the thickness of the Hartmann layer remains unchanged, still scaling as $1 / \mathrm{Ha}$, the slip velocity increases and the velocity gradient in the Hartmann layer decreases as the slip length increases. It has been shown that the pressure drop coefficient in the asymptotic case of a strong magnetic field is reduced by factor $(1+S)$, where $S$ is a new dimensionless parameter, which expresses the slip length to the Hartmann layer thickness ratio.

Second, a fully developed flow in a rectangular duct has been considered in a particular case of hydrodynamic slip at the Hartmann walls and no slip at the side walls. An analytical solution has been obtained (see Eqs. 29) using the standard technique by expanding the velocity and the induced magnetic field as Fourier cosine series in the direction perpendicular to the applied magnetic field. More general case (all walls have the slip) cannot be attacked in the same way because of the limitations of the analytical technique and will require a numerical or asymptotic approach. As shown, the flow demonstrates a core, where the velocity tends to be uniform, while the velocity gradients are confined to the boundary layers. The boundary layers at the walls perpendicular to the magnetic field, as directly seen from the results, still have the thickness scaling as $1 / \mathrm{Ha}$ and thus can be referred to as Hartmann layers. The boundary layers at the side walls are, however, not scaled as $1 / \sqrt{H a}$. They can be significantly thicker than the classic side layers, especially if the slip length is comparable or larger than the thickness of the Hartmann layer. Correspondingly, the core area of uniform velocity in the center of the duct can be significantly smaller compared to the same flow in the no-slip conditions. There are also four regions at the corners where the Hartmann and the side layers overlap. The analysis performed for the fully developed flows in both 1-D and 2-D shows the importance of parameter $S$. Namely, if $S>>1$ (the slip length is much larger than the Hartmann layer thickness), the flow is fully dominated by the hydrodynamic slip while the induced electric currents are small, having almost no effect on the flow. If $S<<1$ (the slip length is much smaller than the Hartmann layer thickness), the slip effect is negligible and the flow can be treated as purely MHD flow. In the case of $S \sim 1$, the slip and the MHD effect are both important.

Third, a time-dependent flow in a rectangular duct in a strong transverse magnetic field has been considered. The 3-D equations have been reduced to a Q2D form via their analytical integration along the magnetic field lines, using the asymptotic representation of the velocity profile obtained earlier in the slip conditions (see Eq. 32). The derived Q2D equations (Eqs. 33 and 34) are, in fact, the extension of the classic equations 
obtained earlier in [15], also known as "SM82" equations. The novelty introduced in the present analysis is the modification of the classic Hartmann braking time, which expresses the characteristic timescale of damping vortical structures due to Joule and viscous dissipation in the Hartmann layers. As shown, the Hartmann braking time is increased by factor $(1+S)$ (see Eq. 35). As an illustration of the slip effect on a Q2D flow, 2-D DNS-like computations are performed for a flow having an "M-shaped" velocity profile. Such a flow becomes unstable and eventually turbulent if the Reynolds number is significantly high. As expected, the flow demonstrates more irregular behavior as parameter $S$ increases.

In conclusion, it may be said that the present theory should be verified experimentally as all experimental evidences of the slip behavior are indirect and no direct quantitative measurements for the slip length have been performed yet in the above mentioned liquid metal flows in contact with ceramics, and also because more complex interfacial boundary conditions (rather than the simple Navier's slip condition used in this study) may be needed. Such an experiment will require a strong magnetic field or/and specially designed hydrophobic surfaces. On the other hand, the present theory have demonstrated that engineering such hydrophobic surfaces can be favorable for fusion applications, such as a DCLL blanket, as their use may result in a dramatic impact on the MHD pressure drop and heat transfer enhancement. There can be some limitations of the theory in case of large Hartmann slip, as it could happen that no uniform core is present when the slip length is large. These limitations have also to be deduced from properly organized experiments or 3-D numerical modeling.

Acknowledgments The study has been performed under DOE grant DE-FG02-86ER52123-A040.

Open Access This article is distributed under the terms of the Creative Commons Attribution Noncommercial License which permits any noncommercial use, distribution, and reproduction in any medium, provided the original author(s) and source are credited.

\section{References}

1. Darhuber, A.A., Troian, S.M.: Principles of microfluidic actuation by modulation of surface stresses. Annu. Rev. Fluid Mech. 37, 425-455 (2005)

2. Blake, T.D.: Slip between a liquid and a solid: D.M. Tolstoi's (1952) theory reconsidered. Colloids Surf. 47, 135-145 (1990)

3. Cho, J.-H.J., Law, B.M., Rieutord, F.: Probing nanoscale dipole-dipole interactions by electric force microscopy. Phys. Rev. Lett. 92, 166101 (2004)

4. Priezjev, N.V., Darhuber, A.A., Troian, S.M.: Slip behavior in liquid films on surfaces of patterned wettability: Comparison between continuum and molecular dynamic simulations. Phys. Rev. E 71, 041608 (2005)

5. Joly, L., Ybert, C., Bocquet, L.: Probing the nanohydrodynamic at solid-liquid interfaces using thermal motion. Phys. Rev. Lett. 96, 046101 (2006)

6. Lauga, E., Brenner, M., Stone, H.: Handbook of Experimental Fluid Dynamics. Springer, New York (2007)

7. Choi, C.-H., Kim, C.-J.: Large slip of aqueous liquid flow over a nanoengineered superhydrophobic surface. Phys. Rev. Lett. 96, 066001 (2006)

8. Pint, B.A., More, K.L., Meyer, H.M., Distefano, J.R.: Recent progress addressing compatibility issues relevant to fusion environments. Fusion Sci. Technol. 47, 851-855 (2005)

9. Morley, N.B., Medina, A., Abdou, M.A.: Measurements of specific electrical contact resistance between SiC and lead-lithium eutectic alloy, TOFE 18, San Francisco, September 28-October 2, Book of Abstracts, P2.66 (2008)

10. Changho, P., Kazuyuki, N., Yamamoto, Y., Konishi, S.: Compatibility of materials for advanced blanket with liquid LiPb, TOFE 18, San Francisco, September 28-October 2, Book of Abstracts, P1.67 (2008)

11. Smolentsev, S., Moreau, R., Abdou, M.: Characterization of key magnetohydrodynamic phenomena in PbLi flows for the US DCLL blanket. Fusion Eng. Des. 83, 771-783 (2008)

12. Moreau, R.: Magnetohydrodynamics. Kluwer, Dordrecht (1990)

13. Hartmann, J.: Hg-dynamics. I. Theory of the laminar flow of an electrically conductive liquid in a homogeneous magnetic field. Math-Fys Medd 15(6), 1-28 (1937)

14. Hunt, J.C.R.: Magnetohydrodynamic flow in rectangular ducts. J. Fluid Mech. 21, 577-590 (1965)

15. Sommeria, J., Moreau, R.: Why, how, and when, MHD turbulence becomes two-dimensional. J. Fluid Mech. 118, 507$518(1982)$

16. Müller, U., Bühler, L.: Magnetofluiddynamics in Channels and Containers. Springer, Berlin (2001)

17. Bühler, L.: Instabilities in quasi-two-dimensional magnetohydrodynamic flow. J. Fluid Mech. 326, 125-150 (1996)

18. Smolentsev, S., Moreau, R.: Modeling quasi-two-dimensional turbulence in MHD duct flows, CTR, Stanford University. Proceedings of the 2006 Summer Program, pp. 419-430 (2006)

19. Messadek, K., Moreau, R.: An experimental investigation of MHD quasi-two-dimensional turbulent shear flows. J. Fluid Mech. 456, 137-159 (2002) 